\title{
INDEX OF VOLUME XXXII
}

Adams, E. P. See Reviews, under Jeans.

Ballantine, J. P. On a certain Functional Condition, 153.

Bateman, H. See Reviews, under McLaren.

BeLL, E. T. A Ray of Numerical Functions of $r$ Arguments, 341.

- On a Fundamental Formula in the Theory of Class-Number Relations 682.

See Reviews, under Dickson.

Bennett, A. A. Consecutive Quadratic Residues, 283.

Bernstein, B. A. On the Serial Relation in Boolean Algebras, 523.

- A General Theory of Representation of Finite Operations and Relations, 533.

- Reports of Meetings of the San Francisco Section: October Meeting 97; April Meeting, 212; June Meeting, 417.

See Reviews, under Whitehead.

Birkhoff, G. D. See Veblen, O.

Bliss, See Veblen, O.

- A Boundary Value Problem in the Calculus of Variations, 317.

- See Reviews, under Vivanti.

Blumberg, H. Properties of Unrestricted Real Functions, 132.

- Note on a Theorem of Kempner concerning Transcendental Numbers, 351.

Brown, B. H. See Reviews, under Baker, Neuberg.

CAjORI, F. See Reviews, under Reymond.

Camp, B. H. See Reviews, under Secrist.

CARLson, E. On the Convergence of Trigonometric Approximations for a Function of Two Variables, 639.

Carmichael, R. D. See Reviews, under Birkhoff.

Carson, J. R. The Heaviside Operational Calculus, 43.

Church, A. On Irredundant Sets of Postulates, 626.

Coolidge, J. L. See Reviews, under Simon.

Cowley, E. B. See Reviews, under Scott.

Crathorne, A. R. See Reviews, under Borel.

Dickson, L. E. All Integral Solutions of $a x^{2}+b x y+c y^{2}=w_{1} w_{2} \cdots w_{n}, 644$.

DodD, E. L. The Convergence of a General Mean of Measurements to the True Value, 282.

- See Reviews, under Day.

Douglas, J. The Transversality Relative to a Surface of $\mathcal{S} F\left(x, y, z, y^{\prime}, z^{\prime}\right) d x$ $=$ Minimum, 669 .

- A Characteristic Property of Minimal Surfaces, 635.

Dresden, A. Some Recent Work in the Calculus of Variations, 475.

- Report of the Thirty-Second Summer Meeting of the American Mathematical Society, 577.

See Reviews, under Pascal, Tonelli. 
Dresden, A., and Richardson, R. G. D. Report of the Thirty-First Summer Meeting of the American Mathematical Society, 1.

Dresden, A. and Stouffer, E. B. The Western Christmas Meeting of the American Mathematical Society, and the Meeting of the Southwestern Section, 101.

DUNKEL, O. The Alternation of Nodes of Linearly Independent Solutions of Second Order Difference Equations, 333.

Eмch, A. See Reviews, under Brill, Schmid.

ErTLINGER, H. J. Note on a Fundamental Lemma concerning the Limit of a Sum, 69.

See Reviews, under Kowalewski, Lovitt.

Forsyth, C. H. See Reviews, under Spillman.

FrEChET, M. The International Conference for the use of Esperanto in Science, 41.

Gehman, H. M. On Irredundant Sets of Postulates, 159.

Glover, J. W. See Reviews, under Loewy.

Graustein, W. C. An Invariant of a General Transformation of Surfaces, 357.

Graves, L. M. Some Problems concerning Measurable Functions, 529.

Gron wall, T. H. See Reviews, under Hayashi.

Hildebrandt, T. H. The Second Ithaca Colloquium, 24.

- The Borel Theorem and its Generalizations, 423.

- See Revirws, under Pringsheim.

Hurwitz, W. A. A Trivial Tauberian Theorem, 77.

JACkson, D. Note on a Problem in Approximation with Auxiliary Conditions, 259.

On a Tensor of the Second Rank in Function Space, 641.

James, G. On the Solution of Higher Degree Algebraic Equations, 162.

KarPINSKI, L. C. See Reviews, under Fettweis.

KELLOGG, O. D. On Some Theorems of Bôcher concerning Isolated Singular Points of Harmonic Functions, 664.

- Recent Progress with the Dirichlet Problem, 601.

- See Reviews, under Hurwitz.

Koopman, B. O. On the Extension of a Method of Briot and Bouquet for the Reduction of Singular Points, 674.

Kormes, M. On the Functional Equation $f(x+y)=f(x)+f(y), 689$.

Kovarik, A. F. See Reviews, under Birtwistle.

KRYLOFF, N. Approximate Solutions of a System of Differential Equations of Mathematical Physics by Least Squares, 346.

LANE, E. P. Wilczynski's and Fubini's Canonical Systems of Differential Equations, 365.

LANGER, R. E., and TAMarkin, J. D. A Notion of Uniform Integrability. 335.

LANGFord, C. H. On Quantifiers for General Propositions, 694.

Lefschetz, S. See Reviews, under Jung.

LeHMer, D. H. A List of Errors in Tables of the Pell Equation, 545.

- Note on the Mersenne Number $2^{139}-1,522$.

LeHMER, D. N. On the Construction of Factor Stencils, 149. 
See Reviews, under Cunningham.

Light, G. H. See Reviews, under Sullivan.

Longley, W. R. See Reviews, under Hort.

Macinnes, C. R. See Reviews, under Carey.

Miller, G. A. The Transformation of a Regular Group into its Conjoint, 631 .

See Reviews, under Fueter, Wieleitner.

Moore, C. L. E. See Reviews, under Cartan.

MOoRE, R. L. Covering Theorems, 275.

A Connected and Regular Point Set which Contains No Arc, 331.

Moore, T. W. Notes on the Rational Plane Cubic Curve, 269.

Nelson, A. L. The Conditions for a Fixed Point in Projective Differential Geometry, 165.

Nelson, C. A. Note on Rational Plane Cubics, 71.

Nowlan, F. S. Representation of Integers by Certain Ternary Cubic Forms, 374 .

Page, L. See Reviews, under De Donder.

Phillips, H. B. See Reviews, under Biezeno.

PIERCE, T.A. An Approximation to the Least Root of a Cubic Equation with Application to the Determination of Units in Pure Cubic Fields, 263.

Pierpont, J. Some Modern Views of Space, 225.

- Note on Horospheres, 525.

RAYNOR, G. E. Isolated Singular Points of Harmonic Functions, 537.

- On the Integro-Differential Equation of the Bôcher Type in ThreeSpace, 654 .

Reynolds, C. N. See Reviews, under De Donder, Dupont, Joly, Neumann.

Richardson, R. G. D. Report of the Secretary to the Council for the Years 1921-1925, 203.

- Reports of Meetings of the American Mathematical Society: October Meeting in New York, 31; Thirty-Second Annual Meeting, 115; February Meeting in New York, 193; April Meeting in Chicago, 215; May Meeting in New York, 305.

See DresDen, A.

Rietz, H. L. See Reviews, under Coolidge, Tschuprow.

Rowe, C. H. Note on a Pair of Properties which Characterize Continuous Functions, 285.

Shaw, J. B. See Reviews, under Netto.

Sierpinski, W. Nuclear Points in the Theory of Abstract Sets, 649.

Sisam, C. H. See Reviews, under Adams, White.

SmaIl, L. L. See Reviews, under Schwatt.

Smith, D. E. See Reviews, under Andoyer, Maroger, Nicomachus, Quemper de Lanascol, Weeks.

SNyDER, V. See Reviews, under Enriques.

Stouffer, E. B. See Dresden, A.

TAMarkin, J. D. See Langer, R. E. 
Tamarkin, J. D., and Wilder, C. E. Note on the Second Law of the Mean for Integrals, 151.

Uhler, H. S. See Reviews, under Madelung, Tuttle.

VAN Vleck, E. B. The Chauvenet Prize for Mathematical Exposition, 40.

Veblen, O., Birkhoff, G. D., and Bliss, G. A. The Colloquium Publications, 100.

Weisner, L. Generalization of Lagrange's Theorem, 629.

Whittemore, J. K. See Reviews, under Lewent.

Whyburn, G. T. Two-Way Continuous Curves, 659.

Wilder, C. E. See TAmarkin, J. D.

WILDER, R. L. A Theorem on Connected Point Sets which are Connected im Kleinen, 338.

Williams, K. P. See Reviews, under Fladt.

Wilson, E. B. See Reviews, under Hewes.

WiLson, W. A. A Curious Irreducible Continuum, 679.

- See Reviews, under Kamke.

Wong, B. C. On the Correspondence between Space Sextic Curves and Plane Quartics in Four-Space, 156.

\section{REVIEWS}

Adams, O. S. Elliptic Functions Applied to Conformal World Maps, C. H. Sisam, 172.

Andoyer, H., et Humbert, P. Histoire des Sciences en France, première partie (Histoire de la Nation Française, rédigée par G. Hanotaux, tome XIV, partie 1), D. E. SmITH, 387.

Baker, H. F. Principles of Geometry, volume III, B. H. BRown, 173.

Biezeno, C. B., and Burgers, J. M. Proceedings of the First International Congress for Applied Mechanics, H. B. Phillips, 563.

Birkhoff, G. D. The Origin, Nature and Influence of Relativity, R. D. CARMICHAEL, 705.

Birtwistle, G. The Principles of Thermodynamics, A. F. Kovarik, 395.

Borel, E. Traité du Calcul des Probabilités, tome I, fascicule I, et tome II, fascicule III, A. R. Crathorne, 398.

Bricard, R. See Quemper de Lanascol, A.

Brill, A. Vorlesungen über Ebene Algebraische Kurven und Algebraische Funktionen, A. Емсн, 292.

Burgers, J. M. See Biezeno, C. B.

Carey, F. S., and Proudman, J. The Elements of Mechanics, C. R. MACINNES, 172.

Cartan, E. La Géométrie des Espaces de Riemann, C. L.E. Moore, 717.

Coolidge, J. L. An Introduction to Mathematical Probability, H. L. RIETZ, 83.

Courant, R. See Hurwitz, A.

Cunningham, A. J. C. Binomial Factorizations, volume V, D. N. LeHMER, 719.

Cunningham, A. J. C., and Woodall, H. J. Factorization of $y^{n} \pm 1$, D. N. LEHMER, 720. 
Day, E. P. Statistical Analysis, E. L. Dodd, 564.

De Donder, $T$. Théorie Mathématique de l'Electricité, première partie, L. PAGE, 174.

- Introduction à la Gravifique Einsteinienne, C. N. REynoLds, 563.

Dickson, L. E. Modern Algebraic Theories, E. T. BeLL, 707.

D'Ooge, M. L. See Nicomachus.

Dupont, P. La Mécanique Nouvelle, démontrée par les Principes Classiques, C. N. ReYnolds, 296.

Enriques, F. Gli Elementi d'Euclide e la Critica Antica e Moderna, libri I-IV, V. SNYDER, 398.

Euclid. See Enriques, F.

Fettweis, E. Wie man einstens Rechnete, L. C. KARPINSKI, 399.

Fladt, K. See Simon, M.

- Unendliche Reihen, K. P. Williams, 171.

Fueter, R. Synthetische Zahlentheorie (zweite Auflage), G. A. Miller, 400 .

Hanotaux, G. See Andoyer, H.

Hayashi, K. Sieben- und Mehrstellige Tafeln der Kreis- und Hyperbelfunktionen und deren Produkte sowie der Gammafunktion, nebst einem Anhang: Interpolations- und sonstige Formeln, T. H. GRON WALL, 716.

Hewes, L. I., and Seward, H. L. Design of Diagrams for Engineering Formulas and the Theory of Nomography, E. B. WrLson, 295.

Hort, W. Die Differentialgleichungen des Ingenieurs (zweite Auflage), W. R. LONGLEY, 87.

Humbert, P. See Andoyer, $H$.

Hurwitz, A. Vorlesungen über Allgemeine Funktionentheorie und Elliptische Funktionen, ergänzt durch einen Abschnitt über Geometrische Funktionentheorie von R. Courant (zweite Auflage), O. D. KELLOGG, 288.

International Congress of Applied Mechanics. See Biezeno, C. B.

Jeans, J, H. Atomicity and Quanta, E. P. AdAMs, 720.

Joly, G. La Relativité Générale, Théorie des Axes Mobiles, C. N. REYNOLDs, 401.

Jones, R. See Lewent, L.

Jung, H. W. E. Algebraische Flächen, S. LefSChetz, 718.

Kamke, E. Das Lebesguesche Integral, W. A. Wilson, 296.

Karpinski, L. C. See Nicomachus.

Kowalewski, G. Einführung in die Determinantentheorie (zweite Auflage), H. J. EtTlingeR, 171.

Lewent, L. Conformal Representation. Translated by R. Jones and D. H. Williams, J. K. Whittemore, 400.

Loewy, A. Versicherungsmathematik (vierte Auflage), J.W.GLover, 714.

Lovitt, W. V. Linear Integral Equations, H. J. ETTLINGER, 169.

McLaren, S. B. Scientific Papers, H. Bateman, 175.

Madelung, E. Die Mathematischen Hilfsmittel des Physikers (zweite Auflage), H. S. UhLER, 718. 
Maroger, A. Le Problème de Pappus et ses Cents Premières Solutions, avec une Préface de P. Montel, D. E. SMith, 715.

Montel, P. See Maroger, A.

Netto, E. Die Determinanten (zweite Auflage), J. B. SHaw, 401.

Neuberg, J. Bibliographie des Triangles Spéciaux, B. H. Brown, 86.

Neumann, E. R. Vorlesungen zur Einführung in die Relativitätstheorie, C. N. Reynolds, 401.

Nicomachus. The Arithmetic of Nicomachus. Translated by M. L. D'Ooge, with Studies in Greek Arithmetic by F. E. Robbins and L. C. Karpinski, D. E. Smrth, 557.

Pascal, E. Lezioni di Calcolo Infinitesimale (quinta edizione), parte II, A. Dresden, 171.

Pringsheim, A. Vorlesungen über Zahlen- und Funktionenlehre, zweiter Band, erste Abteilung, T. H. Hildebrandt, 551.

Proudman, J. See Carey, F. S.

Quemper de Lanascol, A. Géométrie du Compas, avec une Préface de R. Bricard, D. E. Sмith, 396.

Reymond, A. Histoire des Sciences Exactes et Naturelles dans l'Antiquité Gréco-Romaine, F. CAJORI, 556.

Robbins, F. E. See Nicomachus.

Russell, B. See Whitehead, A. N.

Satterly, J. See Tuttle, L.

Schmid, T. Maschinenbauliche Beispiele für Konstruktionsübungen zur Darstellenden Geometrie, A. Емсн, 393.

Schwatt, I. J. An Introduction to the Operations with Series, L. L. SmaIL, 88.

Scott, C. A. An Introductory Account of certain Modern Ideas and Methods in Plane Analytical Geometry (second edition), E. B. COWLEY, 295.

Secrist, H. An Introduction to Statistical Methods (revised edition), B. H. CAMP, 389.

Seward, H. L. See Hewes, L. I.

Simon, M. Nichteuklidische Geometrie, herausgegeben von K. Fladt, J. L. COOLIDGe, 394.

Spillman, W. J. The Law of Diminishing Returns, C. H. Forsyth, 86. Sullivan, J. W. N. A History of Mathematics in Europe, G. H. LIGHT, 88. Tonelli, L. Fondamenti di Calcolo delle Variazioni, volume 1 e volume 2, A. Dresden, 381.

Tschuprow, A. A. Grundbegriffe und Grundprobleme der Korrelationstheorie, H. L. RIETz, 561.

Tuttle, L., and Satterly, J. The Theory of Measurements, H. S. UhLER, 562.

Vivanti, G. Elementi del Calcolo delle Variazioni, G. A. BLIss, 392.

Weeks, R. Boy's Own Arithmetic, D. E. Smith, 175.

White, H. S. Plane Curves of the Third Order, C. H. Sisam, 555.

Whitehead, A. N., and Russell, B. Principia Mathematica (second edition), volume 1, B. A. Bernstein, 711. 
Wieleitner, H. Der Gegenstand der Mathematik im Lichte ihrer Entwicklung, G. A. MiLleR, 397.

Williams, D. H. See Lewent, L.

Woodall, H. J. See Cunningham, A. J. C.

Errata, 760.

Index of Volume XXXII, 747.

New Publications, 93, 187, 301, 406, 571, 727.

Notes, 89, 176, 297, 402, 565, 721.

Papers Read Before the Society and Subsequently Published, ThirtyFifth Annual List of, 731.

\section{Notes and Other Items}

Academies, Associations, Congresses, and Societies:

American Mathematical Society: Annual Meeting, 5, 216, 565, 580; Auditors' Report, 118; Budget, 118; Bulletin, 5, 32, 565; By-Laws, 721; Colloquia, 1, 100, 119, 119, 216; Committee on Colloquia, 119, 216; on Nominations, 5, 216; on Policy, 579; on Printing, 5; Endowment Fund, 6, 119; Election of Officers, 118; Gibbs Lecture, 102, 119, 565; Historical Note, 721; Librarian's Report, 118; List of Officers and Members, 721; New Members Admitted, 3, 31, 116, 215, 578; Prize Funds, 721; Representatives on Council of American Association for the Advancement of Science, 119, on National Research Council, 119; San Francisco Section, 97, 417; Statistics, 117; Summer Meetings, 5, 580; Transactions, 32, 89, 402, 565, 565, 721; Treasurer's Report, 118, 721.

Associations for the Advancement of Science: American, 101, 119, 177, 178, 565; British, 89, 722; French, 89, 297.

American Physical Society, 101, 178; American Society of Mechan ical Engineers, 567; Belgian Academy, 90, 177, 402, 403, 567; Berlin Mathematical Society, 178; Calcutta Mathematical Society, 90; College Entrance Examination Board, 566; Danish Academy, 297; Edinburgh Mathematical Society, 402; Franklin Institute, 403; Institute of International Education, 723; International Astronomical Union, 89; International Congress for Applied Mechanics, 566; International Mathematical Congress, 722; International Research Council, 89, 402; London Mathematical Society, 176, 177; Mathematical Association of America, 40, 41; Naples Academy, 567; National Academy of Sciences, 32, 176, 402; National Research Council, 119; National Research Endowment, 176; New York Academy of Sciences, 722; Paris Academy of Sciences, 177; Physico-Mathematical Society of Kazan, 177; Reale Accademia dei Lincei, 90; Rockefeller Foundation, General Education Board, 32; Royal Academy of Sweden, 297; Royal Astronomical Society, 178; Royal Society of Edinburgh, 567; Royal Society of London, 178; Russian Academy, 90; Società Italiana delle Scienze, 177; Société Mathématique de Belgique, 566; Verein Deutscher Ingenieure, 402. 
Books, Announcement of New, 176, 176, 177, 402, 566, 566.

Doctorates in Mathematics, American, 179.

Fellowships: American Field Service, 723; Guggenheim, 178; German Student Exchange, 723; National Research, 568.

Journals :

American Journal of Mathematics, 89, 176, 297, 566, 721; Annals of Mathematics, 89, 176, 297, 566, 722; Bulletin of the American Mathematical Society, 5, 32, 565; Journal of the Faculty of Science, Imperial University of Tokyo, 566; Journal of the London Mathematical Society, 176; Transactions of the American Mathematical Society, 32, 89, 402, 565, 565, 721 .

Papers and Communications Presented to the Society, Authors:

Adams, C. R., 197; Adams, R. B., 309; Agnew, R. P., 19; Aldrich, G. P., 597; Ayres, W. L., 37, 131, 307, 308.

Babb, M. J., 38; Babcock, R. W., 223; Ballantine, J. P., 195, 196, 196; Barter, J. D., 214, 214; Bell, E. T., 23, 124, 124, 125, 125, 125, $125,417,418,418,577$; Bender, H. A., 586; Bennett, A. A., 9, 23, 33, Bennett, T., 112; Bernstein, B. A., 418; Blichfeldt, H.F., 99; Born, M., 129; Brenke, W. C., 105; Brinkmann, H. W., 10, 10, 10, 10, 202; Brown, E. P., 120; Brown, J. F., 418; Buchanan, D., 418, 419.

Cajori, F., 98, 212; Camp, B. H., 9; Camp, C. C., 106, 222, 589, Campbell, A. D., 8, 12, 111, 111; Campbell, J. W., 598; Carman; M. G., 308; Carmichael, R. D., 111, 111, 112, 113; Carpenter, A. F.; 212, 419, 419; Carver, W. B., 7; Chittenden, E. W., 110, 577; Cleveland, C. M., 109, 201, 311, 420; Coble, A. B., 113; Court, N. A., 113, 583; Cramlet, C. M., 212, 419.

Dantzig, T., 314; Davis, H. T., 107, 221; Dederick, L. S., 105, 105; Dickson, L. E., 39, 222, 587; Dines, L. L., 22, 22, 420, 420, 420; Dodd, E. L., 104, 198; Dresden, A., 216; Dunkel, O., 105.

Edington, W. E., 109; Eiesland, J., 11, 12; Eisenhart, L. P., 28, 197; Emch, A., 313, 583; Ettlinger, H. J., 18, 34; 220, 593, 593, 594, 594, 594, 598; Evans, G. C., 599.

Farnum, F., 98; Feinler, F. J., 126, 126; Fields, J. C., 102, 123, 123; Fite, W. B., 121; Foraker, F. A., 582; Ford, L. R., 19, 113; Fort, T., 121; Foster, M. C., 122, 313; Franklin, P., 37, 37, 128, 198; Frink, O., 126, 201.

Gage, W. H., 419; Garabedian, C. A., 11, 11, 590; Gehman, H. M., 33, 34, 39, 199, 199, 199, 218, 310, 420, 591, 591; Georges, J. S., 314; Glenn, O. E., 20, 315, 586; Goff, J. A., 113; Graves, L. M., 14, 224, 311; Griffiths, L. W., 99; Gronwall, T. H., 23, 36, 36, 36, 127, 127, 195, 316, 316. 
Haskell, M. W., 99; Hazlett, O. C., 108, 114, 588; Hedrick, E. R., 15, 16, 213; Hildebrandt, T. H., 102; Hill, L. S., 595, 596; Hille, C. E., 17, 196, 196, 314; Hollcroft, 7, 197, 306, 582; Hotelling, H., 97, 98, 98, 98; Huntington, E. V., 104.

Ingold, L., 112, 112; Ingraham, M. H., 109, 589.

Jackson, D., 29, 39, 106, 223, 600; Johnston, C. I., 418.

Karpinski, L. C., 127; Kellogg, O. D., 19, 33, 120, 316, 590; Kline, J. R., 114; Koopman, B. O., 128, 128, 592; Kormes, M., 33, 130, 308; Kryloff, N., 106.

LaMer, V. K., 316; Lane, E. P., 218, 219, 598; Langer, R. E., 120, 309; Langford, C. H., 124, 202, 308; Lefschetz, S., 195; Lehmer, D. H., 421, 421; Lehmer, D. N., 99, 421; Levy, H., 312, 312, 313; Lubben, R. G., 13, 14, 14, 109, 114, 200, 200, 310.

McAlister, E. H., 422; McDonald, J. H., 99, 213, 213, 214; McGiffert, J., 121; MacDuffee, C. C., 587; Manning, H. P., 32; Maria, A. J., 313; Mathews, R. M., 219; Mathewson, L. C., 198; Merriman, G. M., 223; Messick, C. A., 103; Michal, A. D., 122, 123, 584, 595; Miller, G. A., 20, 585, 585; Miller, N., 597; Mitchell, H. H., 305; Moore, C. L. E., 8, 583; Moore, C. N., 16, 224; Moore, E. H., 224; Moore, R. L., 13, 13, 110, 198, 218; Moore, T. W., 305; Morley, F., 306; Morse, H. M., 17, 595; Mullings, M. E., 589; Murnaghan, F. D., 12, 307, 590; Murray, F. H., 8.

Nassau, J. J., 224; Neelley, J. H., 584; Neikirk, L. I., 421; Nelson, C. A., 12; Niemytski, V. W., 597; Nowlan, F. S., 107.

Olson, J. L., 585, 585.

Patterson, B. C., 306; Perkins, F. W., 127, 128; Pfeiffer, G. A., 202; Pierce, T. A., 107; Pierpont, J., 102, 306; Poor, C. L., 193, 194; Poor, V. C., 131, 131; Porter, M. B., 15, 16; Putnam, T. M., 99.

Rainich, G. Y., 8, 9, 32, 126, 129, 194, 307, 307, 582; Raynor, G. E., 35, 600; Rees, W. A., 593; Richardson, R. G. D., 16; Richmond, D. E., 130; Rider, P. R., 104, 221, 590; Ritt, J. F., 201, 201; Robertson, H. P., 312; Robinson, L. B., 316, 590.

Schouten, J. A., 18; Schwartz, P. D., 315; Schwatt, I. J., 33; Sharpe, F. R., 7; Shohat, J. A., 18, 104, 220, 585 ; Sierpinski, W., 597; Simmons, H. A., 221; Sisam, C. H., 108; Smail, L. L., 19; Smith, D. E., 120; Smith, H. L., 223, 223; Smith, P. A., 195; Smith, R. G., 220; Snyder, V., 583; Stark, M. E., 129; Starke, E. P., 315; Stone, M. H., 38, 38, 130, 595 ; Stouffer, E. B., 108, 220, 220; Sturdivant, J. H., 592; Swingle, P. M., 110.

Tamarkin, J., 17, 18, 120, 120, 309, 309, 309, 600; Taylor, J. H., 194; Thomas, J. M., 122, 597; Thomas, T. Y., 12, 38, 129, 584; Turner, J. S., 108, 219. 
Vandiver, H. S., 21, 21, 21, 21, 22, 125, 309, 310, 587, 587, 588; Vinogradov, I. M., 596.

Wahlin, G. E., 219, 588; Walker, E., 124; Walsh, J. L., 35, 35, 198, 599; Ward, L. E., 221; Weaver, J. H., 581; Weida, F. M., 122; Weisner, L., 7, 20; Pell Wheeler, A., 121; Whyburn, G. T., 109, 200, 217, 310, 311, 421, 422, 592, 592; Whyburn, W. M., 596; Wiener, N., 37, 129; Wilder, C. E., 18, 120, 600; Wilder, R. L., 34, 34, 123, 217, 217, 218, 591; Williams, W. L. G., 20; Williamson, J., 222; Wilson, N. R., 107; Wilson, W. A., 15, 15, 35; Wong, B. C., 214.

Young, J. W., 584, 588, 589.

Personal Notes:

Adams, W. S. 568; Agnola, A. dell', 723; Agostini, A., 404; Ahalt, H. C., 299; Aiyar, A., 90; Aitken, A. C., 179; Alderton, N. M., 299; Aldrich, G. P., 186; Alexander, J. W., 404; Ames, L. D., 184, 724; de Amicis, E., 186; Amira, B., 723; Anderson, R. L., 405; Anderton, E. L., 179; Antoine, L., 568; Appell, P., 90, 298; Archibald, R. C., 177, 183; Armstrong, G. N., 92; Arnold, W. C., 299; Arrhenius, A. S., 403.

Babb, M. J., 91, Babcock, R. W., 299; Bagby, L. C., 184; Bailey, L. H., 177; Baillaud, B., 89; Baillaud, J., 298; Baire, R., 298; Ball, W. W. R., 568; Ballantine, J. P., 568; Barnard, R. W., 568; Barr, C. F., 724; Barton, E. H., 186; Barton, S. M., 405; Barus, C., 183; Bateman, H., 722; Beck-Ustni, A., 726; Beckwith, E. R., 184; Behmann, H., 724; Bell, C., 179; Bell, E. T., 119, 404; Bennett, T. L., 179; Berger, E., 726; Bergson, H., 177; Berry, E. M., 724; Berry, W. J., 299; Berwick, W. E. H., 568; Berzolari, L., 91; Bessel-Hagen, E., 91; Betz, A., 724; Bieberbach, L., 90, 403; Bigourdan, G., 179; Birkeland, R., 179; Birkhoff, G. D., 183, 402, 402, 565 ; Bliss, G. A., 41; Bohannan, R. D., 299, 405, 580; Bohr, H., 567; Bohr, N., 90, 403, 567, 567, 722; Bompiani, E., 177, 403; Bond, J. D., 404; Borel, E., 89; Borgman, W., 185; Born, M., 183; Bortolotti, E., 404; Bottomley, J. T., 570; Bouligand, G., 177; Bower, E. C., 725; Bower, J. W., 570; Bowie, W., 568; Tycho Brahe, 297; Brant, L., 404; Breuer, S., 298; Brinkmann, H. W., 179; Bristol, W. A., 185; Brixey, J. C., 185; Brown, O. E., 185; Brun, V., 179; Brusotti, L., 403; Buck, T., 404; Burnam, J. E., 569; Burwell, W. R., 183; Butler, N. M., 566.

Caldonazzo, B., 91; Campbell, A. D., 91; Campbell, J. W., 184; Campbell, W. W., 89; Caris, P. A., 91, 179; Carman, M. G., 179; Cartan, E., 297, 723; Carter, B. E., 570; Cecioni, F., 403; Cerf, G., 298; Chalmers, L., 185; Chapman, S., 177; Charlier, C. V. L., 567; Chase, R. M., 299; Chisini, O., 91; Chittenden, E. W., 91, 565; Choudhury, N. R., 90; Coble, A. B., 723, Cohen, A., 724; Cole, F. N., 183, 300, 580; Conwell, G. M., 569; Coolidge, J. L., 119; Cooper, R. C., 298; Cothern, L., 185; Cottrell, F. G., 402; Cox, E. F., 179; Crawley, E. S., 119; von Crayen, W., 90; Cumming, F., 183; Curtis, H. B., 91, 725; Czuber, E., 405. 
Dantzig, T., 569; Davis, J. E., 569; Deakin, C. S., 90; Debye, P., 723; Dederick, L. S., 569; Denjoy, A., 177, 298; Des Coudres, T., 726; Dewey, R., 185; Dickson, L. E., 176, 178; Dixon, A. L., 177; Doehlemann, K., 300; Donati, L., 176; De Donder, T., 183; Doole, H. P., 726; Drach, J., 177, 183; Dreyer, J. L. E., 726; Dymond, E. G., 179; Dyson, F.W., 722 .

Eddington, A. S., 403, 722; Edmondson, A., 185; Eells, W. C., 569; Einstein, A., 90, 178, 178; Eisenhart, L. P., 723; Enriques, F., 178; Erickson, E. E., 91; Eshleman, J. D., 91; Evans, L., 90; Everett, E. M., 185; Eydoux, D., 177, 179.

Fairon, J., 186, 724; Farnau, E. F., 299; Farnum, F., 570; Fine, H. B., 183; Finzi, B., 404; Fiske, T. S., 566; Flanders, D. A., 184; Flanders, R. L., 184; Ford, L. R., 568; Foster, M. C., 184; Fournier, G., 177; Fox, H., 183; Frattini, J., 570; Fréchet, M., 177, 566; Frege, G., 92; Friedmann, A. A., 186; Frink, O., 568; Fromme, K., 178; Fubini, G., 723; Furch, R., 724 .

Galbraith, M. G., 570; Gale, H. G., 184; Gaunt, J. A., 567; Gehman, H. M., 179, 570; Geppert, H., 91; Giorgi, G., 403; Glassett, F. S., 299; Godeaux, L., 724; Gore, J. D., 185; Gouy, L. G., 405; Grätz, L., 298; Grant, A. A., 185; Graustein, W. C., 177, 567; Gravatt, T. E., 299; Graves, L. M., 404; Gray, A., 186; Green, R. L., 184; Grove, V. G., 179, 299; Guggenbühl, L., 726; Guichard, C., 567; Gwinn, I, 569.

Hadamard, J., 402; Halbwachs, M., 177; Hale, R., 185; Halperin, H., 91; von Hammer, 92; Hammond, E. S., 183; Hardy, G. H., 177, 567; Harkin, D. C., 725; Harshbarger, F., 92; Hartley, R. W., 179, 569; Hasse, H., 90; Hatcher, T. W., 299; Heal, W. E., 186; Heinz, A. A., 405; Henneberg, L., 90; Herr, G. A., 569; Hess, G. W., 569; Hessenberg, G., 185; Hildebrandsson, H. H., 186; Hille, C. E., 568; Hilton, H., 177; Holl, D. L., 92, 180; Holmes, C. T., 183; Hoover, H., 176; Horrocks, H., 298; Horton, G. P., 299; Hoskins, L. M., 92; Howe, H. A., 726; Huber, C. M., 405; Hughes, H. K., 185; Hughes, J. C., 404; Hungington, E. V., 177; Hurst, J. W., 180; Hurwitz, W. A., 725; Hussey, W. J., 726.

Ince, E. L., 568; Ingham, A. E., 724; Ingraham, M. H., 183.

Jackson, D., 566; Jacobson, S., 180; Jaffe, G., 179; James, G., 404; Jensen, J. L. W. V., 92; Johnson, M. I., 725; Johnson, R. A., 569; Jones, T. D., 183; Jordan, H. E., 404; Jung, G., 300.

Kampé de Fériet, J., 91; von Karman, T., 723; Kearney, D. E., 92; Kellogg, V., 402; Kerstein, B. H., 92; Klein, F., 178, 723; Knebelman, M. S., 726; Kneser, H., 298; Knopp, K., 404; Koebe, P., 724; Kokomoor, F. W., 405; Kommerell, K., 179; Koopman, B. O., 568; Koschmeder, L., 90; Kovarik, A. F., 569; Krazer, A., 726; Kretschmer, W., 185; Kuhn, H. W., 299 
Lamb, H., 89; Lamson, K. W., 569; Landau, 723; Lane, E. P., 178; Lang, P. R. S., 726; Langer, R. E., 183; Langevin, P., 567; Langmuir, I., 90; Latham, M., 92; von Laue, M., 90; Laura, E., 723; Lebesgue, H., 90; LeChatelier, H., 567; Lehr, A. M. M., 180; Leonard, R., 185; Levy, P., 91; Lewis, A. B., 184; Lewis, W. J., 570; Lichtenstein, L., 403; Lightfoot, N. M., 91; von Lilienthal, R., 179; Lindsey, L., 404; Linehan, P. H., 184; Linfield, B. Z., 569; Littlewood, J. E., 177; Lorentz, H. A., 178, 403, 567, 723; Lubben, R. G., 180, 568; Ludlow, H. H., 570; Lufkin, H. M., 185; Lyle, G. A., 725.

McEwen, W. H., 185; McFarland, E. J., 185; MacCreadie, W. T., 570; Machmer, W. L., 569; Maizlish, I., 92; Maltbie, W. H., 186; von Mangoldt, H., 186; Maria, A. J., 180; Marletta, G., 403; Marquis, R. H., 405; Marraine, F. G. G. A., 179; Martin, E. N., 299; Maslow, A., 726; Mason, M., 568; Mathews, R. M., 180, 185, 299; Mayer, W., 724; Meacham, E. D., 184; Meder, A. E., 405; Mendenhall, R. M., 570; Menke, H. E., 185; Menzel, D. H., 92; Merriman, G. M., 568; Michelson, A. A., 184; Michkovitch, V., 177; Mickelson, E. L., 185; Miller, D. C., 178; Miller, J. W., 92; Millikan, R. A., 90, 178, 567, 568; Milloux,R., 568; Mills, C. N., 184; Mitchell, H. H., 177; MittagLeffler, G., 403; Montgomery, A. G., 726; Moore, L. T., 180; Morehead, J. C., 404; Morris, A. V., 299; Morse, H. M., 183, 183; Moulton, F. R., 177; Müller, E., 403; Murray, F. H., 404; Musselman, J. R., 404

Nadori, A., 724; Nagaoka, 89; Neder, L., 724; Nelson, A. L., 92; Neuberg, J., 300; Newlin, R. L., 92; Nichols, H. W., 186; Nipher, F. E., 726; Nörlund, N. E., 567; Nowlan, F. S., 180; Nyswander, J. A., 404.

Olds, G. D., 725; Onnes, H. K., 300; Osgood, W. F., 566; Ostrowski, A., 403; Owens, F. W., 569.

Packer, M. C., 404; Painleve, P., 177; Palatini, A., 723; Papps, P. C. H., 726; Paradiso, L. I., 726; Parisi, F. A., 184; Pattenhausen, B., 726; Patterson, B. C., 569; Peed, M. T., 186; Pepper, E. D., 180, 405; Perrycoste, W. B. C., 90; Phalen, H. R., 725; Phillips, J., 186; Picard, E., 89; Picart, L., 298; Pierce, T. A., 299; Pincherle, S., 567; Planck, M., 298, 402, 403, 567, 724; Plimpton, G. A., 115; Pohlhausen, E., 91, 298; Pouliot, A., 178; Powell, E. W., 185; Pupin, M. I., 177, 298, 568, 723; Putnam, R. G., 299.

Radon, J., 91; Rainich, G. Y., 404; Rankin, W. W., 405; Raymond, H., 297; Reaves, S. W., 184; Rechard, O. H., 725; Rees, P. K., 726; Reich, K., 405; Reidemeister, P., 91; Rethy, M., 186; Ricci, G., 92; Richardson, R. G. D., 183; Richert, D. H., 184; Richmond, D. E., 568; Richmond, H. W., 177; Riddle, G. W., 726; Riesz, M., 568; Rietti, T., 186; Rietz, H. L., 725; Risser, R., 177; Robinson, G. E., 569; Robinson, H. A., 569; Robinson, L. V., 725; Robinson, P. G., 180; Room, T. G., 179; Roos, C. F., 568; Rosati, C., 403; Roth, P,. 405; Rowland, S. A., 725; Russell, W. P., 299; Rutherford, E., 177, 178, 567, 567; Rutt, N. E., 185; Ryno, J., 184. 
Salkover, M., 180; Sannia, G., 403; Sansone, G., 403; Schelkunoff, S. A., 570; Schiller, L., 724; Schlesinger, 89; Schlötzer, A., 724 ; Schmidt, R., 91, 298; Schönberg, E., 724; Schug, H. L., 726; Schur, F., 403; Scorza, O., 723; Scott, C. A., 177; Scott, G. H., 726; Sears, K., 185; Serini, R., 91; Severi, F., 723; Sheffer, I. M., 405; Shenton, W. F., 92; Sherrington, C., 177; Short, J. M., 569; Sibirani, F., 403; Siceloff, L. P., 405; Siegbahn, K. M. G., 178; Simester, J. H., 185; Simmons, H. A., 180, 405; Simon, W. G., 570; Simons, L. G., 180; Sinclair, M. E., 725; de Sitter, W., 89; Skarstedt, M., 180; Smail, L. L., 185, 570; Smith, D. E., 115, 119; Smith, H. L., 92, 725; Smith, P. A., 568; Smith, R. J., 722; Snelling, C. M., 183; Snyder, V., 119; Solt, M.R., 726; Sommerfeld, A., 403, 567, 723; von Sourek, A., 726; Sparks, F. W., 725; Stafford, E. T., 184; Staley, R. C., 185; Stark, C. R., 183; Steen, S. W. P., 179; Stekloff, V. A., 570; Stephens, R. P., 183; Stouffer, E. B., 178, 565; Streyffeler, D. O., 185; Sutton, C., 185; Szegö, G., 91, 700.

Tam, V. A., 180; Tamarkin, J. D., 405; Tanner, J. H., 405; Tappan, A. H., 185; Taylor, H. W., 185; Taylor, J. H., 185, 299; Taylor, J. S., 405; Taylor, W. H., 570; Terami, T., 180; Terracini, A., 403; Thiry, R., 179; Thomas, L. H., 298; Thomas, T. Y., 404; Thurston, H. S., 185; Titchmarsh, E. C., 91; Togliatti, E. G., 403; Torrey, M. M., 185; Townes, S. B., 185; Tracey, J. I., 405; Tricomi, F., 403; Turnbull, H. W., 567; Turner, J. S., 725; Tweedie, C., 186.

Uhler, H. S., 570.

Valiron, G., 177; de la Vallée Poussin, C., 178, 403; Vandiver, H.S., 92; Veblen, O., 119, 176. 183, 402; Ver Eecke, P., 90; Vitali, G., 403; Volterra, V., 403, 722.

Wait, A. H., 726; Waldo, C. A., 726; Walker, B. M., 183; Ward, L. E., 180, 185; Watson, G. N., 177; Weaver, J. H., 299; Weida, F. M., 92; Weisner, L., 568; Welsh, W., 186; Wellstein, J., 298; Wentzel, G., 724; Western, A. E., 177; Weyl, H., 91, 723; Weyrich, R., 568; Pell Wheeler, A., 119; Whitehead, A. N., 178, 568; Whittaker, E. T., 567; Whittemore, J. K., 570; Whyburn, W. M., 184; Wiarda, G., 179; Widder, D. V., 568; Wien, W., 298, 722; Wiener, N., 178; Wilder, R. L., 404; Wilkins, P. D., 185; Williams, H. B., 565; Williams, J. E., 178, 299; Williams, W. L. G., 300; Wilson, A. H., 567; Wilson, E. B., 403; Wilson, R. E., 297; Winger, R. M., 405; Winslow, J. B., 185; Wirtinger, W., 403; Witz, A., 300; Wood, F., 185; Woods, R., 91; Worthington, E. R., 405; Wylie, C. C., 91.

Yeaton, C. H., 300.

Zermelo, E., 724; Zsigmondy, C., 186; Zühlke, P., 568.

Prizes and Medals:

American Association for the Advancement of Science, 178; American Society of Mechanical Engineers, 567; Belgian Academy, 90, 177, 402; Belgian Government, 403; Cambridge University: Winbolt, 90, 
722; Smith, 298; Rayleigh, 298; Mayhew, 567; Chauvenet, 40, 41; Danish Academy, 297; Franklin Institute, 403; Graustein, 567; Lobachevsky, 177; Mahamaya, 90; A. Cressy Morrison, 722; Naples Academy, 567; Nobel, 178; Paris Academy of Sciences, 177; Quebec Government, 178; Reale Accademia dei Lincei, 90; Royal Astronomical Society, 178; Royal Society of Edinburgh, 567; Royal Society of London, 178; Società Italiana delle Scienze, 177.

Universities and Technical Schools:

Cambridge, 90, 298, 567, 568, 722;

Chicago, 180 ;

Columbia, 181;

Minnesota, 182;

Cornell, 181;

Ohio State, 182;

Illinois, 181;

Oxford, 90, 700;

Iowa, 181;

Pennsylvania, 182;

Jerusalem, 723;

Princeton, 183;

Johns Hopkins, 181, 721, 723;

Rutgers, 298;

Stanford, 182;

Massachusetts Institute of Technology, 183;

Michigan, 181;

Texas, 182;

Tokyo, 566;

Wisconsin, 182.

\section{ERRATA-VOLUME XXXII}

F. S. Nowlan, Representation of integers by certain ternary cubic forms.

Page 376, formula (5): the sign of the third term on the right side $\left(5 \alpha_{0}^{2} \alpha_{2}\right)$, and the sign of the seventh term $\left(\alpha_{1}^{3}\right)$ should be plus.

Page 378, lines 11-12: The expression for $F_{1}$ should agree with the corrected form of formula (5), p. 376; on p. 378 the sign of the last term should be plus.

T. H. Hildebrandt, The Borel Theorem and its generalizations.

Page 453, second footnote: omit the last sentence, beginning "Moore's example, however, is . . ". 